\title{
USO E OCUPAÇÃO DO SOLO E SUA ASSOCIAÇÃO COM A TEMPERATURA DA SUPERFÍCIE TERRESTRE NO MUNICÍPIO DE BAIXIO - CE
}

\section{Use and occupation of the soil and its association with the terrestrial surface temperature in the municipality of Baixio - CE}

\author{
Diêgo Lima Crispim \\ dlimacrispim@gmail.com \\ Doutorando em Engenharia Civil Universidade Federal do Pará/Programa de Pós-Graduação
}

Paulo Eduardo Silva Bezerra

Graduado em Engenharia Ambiental e Energias Renováveis (UFRA), Mestrando no Programa de Pós-graduação em Engenharia Civil da Universidade Federal do Pará. pauloeduardoea@gmail.com

Rodrigo Silvano Silva Rodrigues rssr@ufpa.br

Engenheiro Sanitarista e Ambiental (UFPA), Mestre em Engenharia Civil (UFPA) e Doutorando no Programa de Pós-graduação em Engenharia Civil (UFPA).

Artur Sales de Abreu Vieira a-sales@hotmail.com

Engenheiro Sanitarista e Ambiental (UFPA), Mestre no Programa de Pós-graduação em Engenharia Civil da Universidade Federal do Pará

Fábio Venilson de Sousa Pereira Analista Ambiental da Prefeitura Municipal de Santa Luzia do Pará. fabiorootssousa@gmail.com

Lindemberg Lima Fernandes Doutor em Desenvolvimento Sustentável do Trópico Úmido Professor da Faculdade de Engenharia Sanitária e Ambiental e da Pós-graduação em Engenharia Civil da Universidade Federal do Pará lberge@ufpa.br

RESUMO: Este trabalho teve como objetivo analisar as modificações no uso e ocupação do solo e verificar sua associação com a temperatura da superfície terrestre do município de Baixio - CE no intervalo de 1986 a 2016. Neste estudo foram empregadas as bandas 3, 4 e 5 do sensor TM/Landsat 5 e das bandas 4, 5 e 6 do OLI/Landsat 8 para observar as alterações no uso do solo. Para estimar a temperatura da superfície terrestre (TST) foram utilizadas duas imagens multiespectrais (bandas do infravermelho termal), correspondente 6 e 10, com resolução de $30 \mathrm{~m}$, dos satélites Landsat 5 e 8, por meio dos sensores TIRS (Thermal Infrared Sensor) para os anos de 1986, 1996, 2006 e 2016. Para classificação do uso do solo foi aplicado à técnica da Máxima Verossimilhança (MAXVER). Os resultados indicam que no decorrer do período pesquisado as classes de uso e ocupação do solo, a saber, corpo hídrico e floresta tiveram uma redução entre os anos de 2006 e 2016. Os resultados mostram um crescimento contínuo da classe de área antrópica no período analisado. A temperatura da superfície terrestre no município de Baixio apresentou um aumento significativo. Os anos com as maiores médias registradas foram 2006 e 2016, porém, com maior destaque em 2016, em que a predominância da temperatura ficou entre os intervalos de 36 a $40{ }^{\circ} \mathrm{C}$ e 40 e $42{ }^{\circ} \mathrm{C}$.

Palavras-chave: Landsat; Cobertura florestal; Sensoriamento remoto; Uso e ocupação da terra. 
USO E OCUPAÇÃO DO SOLO E SUA ASSOCIAÇÃO

COM A TEMPERATURA DA SUPERFİCIE

TERRESTRE NO MUNICÍPIO DE BAIXIO - CE

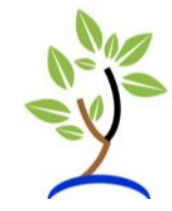

ABSTRACT: The objective of this work was to analyze the changes in land use and occupation and to verify its association with the surface temperature of the municipality of Baixio $-\mathrm{CE}$ in the period from 1986 to 2016. In this study used the bands 3, 4 and 5 of the sensor TM / Landsat 5 and the bands 4, 5 e 6 of the sensor OLI / Landsat 8 to observe changes in soil use. For analyze surface temperature variation used two spatial infrared (VHF) images, 6 and 10, with $30 \mathrm{~m}$ resolution, of the Landsat 5 and 8 satellites were used to estimate the surface temperature (TST) using TIRS (Thermal Infrared Sensor) for years 1986, 1996, 2006 and 2016. For the classification of soil use was applied to the Maximum Likelihood (MAXVER) technique. The results indicate that during the period studied the classes of land use and occupation, namely, water and forest had a reduction between the years 2006 and 2016. The results indicate a continuous growth of anthropic area class in the observed period. The temperature of the terrestrial surface in the municipality of Baixio presented a significant increase in the observed period. The years with the highest averages recorded were 2006 and 2016, however, with a greater emphasis in 2016, where the predominance of temperatures was between the intervals of 36 to $40^{\circ} \mathrm{C}$ and 40 and $42^{\circ} \mathrm{C}$.

Keywords: Landsat; Forest cover, Remote sensing, Occupation and land use.

\section{INTRODUÇÃO}

Segundo Mashiki e Campos (2013) o desenvolvimento das geotecnologias, das informações de sensoriamento remoto e a melhoria nas resoluções das imagens de satélite na atualidade, possibilita a obtenção de vários dados do espaço físico. Assim, um dos dados englobados nas imagens de satélite é a temperatura da superfície terrestre, conseguida por meio da captação da energia eletromagnética irradiada por algum elemento ou objeto.

As técnicas de sensoriamento remoto apresentam-se como um instrumento de grande relevância no acompanhamento de vários fenômenos meteorológicos e climatológicos (FERREIRA et al., 2017), e ambientais (BEZERRA et al., 2014), proporcionando um auxílio nas estimações de tempo e melhor compreensão das alterações climáticas, além servir de suporte na elaboração do planejamento agroecológico (BEZERRA et al., 2014).

Além disso, outro notável benefício do sensoriamento remoto consiste no mapeamento de extensas e pequenas áreas com diversas formas de resoluções temporais e espaciais (PONZONI et al., 2012; FERREIRA, PEREIRA FILHO, 2009).

O sensoriamento remoto proporciona uma rápida aquisição de informações, que frequentemente estão disponíveis de forma grátis em algumas imagens de satélite em base de dados governamentais e não governamentais (MASHIKI; CAMPOS, 2013). Assim, esta técnica notabiliza-se por propiciar a visualização de uma configuração espacial de distintas escalas, assim como proporciona informações do infravermelho termal (FLORENZANO, 2011).

Esta ferramenta auxiliar no entendimento da atuação de variáveis climáticas, a título de exemplo, temperatura de superfície, fluxo de calor, entre outras, tornando viável a monitoração do espaço em escala temporal dos recursos hídricos e o uso e ocupação do solo, em distintos biomas (SILVA et al., 2015). As geotecnologias possibilitaram observações e estudos mais precisos dos fenômenos naturais que acontecem na superfície terrestre, assim como as diversas formas de expansão do uso e ocupação do solo por meio das ações antrópicas (SILVA, 2015). 
USO E OCUPAÇÃO DO SOLO E SUA ASSOCIAÇÃO

COM A TEMPERATURA DA SUPERFİCIE

TERRESTRE NO MUNICÍPIO DE BAIXIO - CE

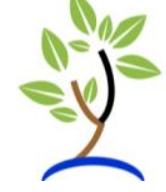

As atividades antrópicas têm ocasionado intensos impactos nas paisagens do ambiente natural por meio do forte processo de modificação das áreas naturais por várias formas de uso e ocupação do solo e da redução da cobertura das áreas florestadas (MATSUSHITA et al., 2006). Assim, vários elementos estão diretamente associados a esta ação, podendo interferir na qualidade e acessibilidade dos recursos naturais, além de prejudicar a biodiversidade em extensas áreas da terra (MENDOZA et al., 2011).

Assim, a alteração no uso do solo são uns dos principais causadores dos impactos ambientais, destacando as alterações da temperatura terrestre, mostrando uma interferência no solo, vegetação e na paisagem local (PEREIRA et al., 2012). Um destes fatores é o uso intenso de áreas para atividades agrícola e pecuária, bastante usual no território do semiárido brasileiro (COELHO et al., 2014). Este tipo de atividade pode ocasionar a diminuição da biodiversidade, redução na fertilidade do solo e o aumento de processos de erosão (VANZELA et al., 2010).

Nesta perspectiva, o uso das técnicas de geoprocessamento e sensoriamento remoto são de suma importância para a análise da Temperatura da Superfície Terrestre (TST) e na dinâmica do uso do solo com o auxílio de imagens de satélites. Segundo Almeida et al. (2015) com o uso das imagens de satélites é possível monitorar a dinâmica do uso do solo em relação a TST. Neste contexto, este trabalho tem como objetivo analisar as modificações no uso e ocupação do solo e verificar seus impactos na temperatura da superfície do território do município de Baixio - CE no intervalo de 1986 a 2016.

\section{MATERIAL E MÉTODOS}

\section{Área de estudo}

O estudo foi realizado no município de Baixio, localizado na região Nordeste do Brasil, mas especificamente no estado do Ceará (Figura 1). Trata-se de um município com uma área de unidade territorial de $146,44 \mathrm{~km}^{2}$, com uma densidade demográfica de $41,15 \mathrm{~km}^{2}$, para uma população de 6.026 , dos quais 3.304 habitantes residem na zona urbana e 2.722 na zona rural (IPECE, 2013; IBGE, 2010). 


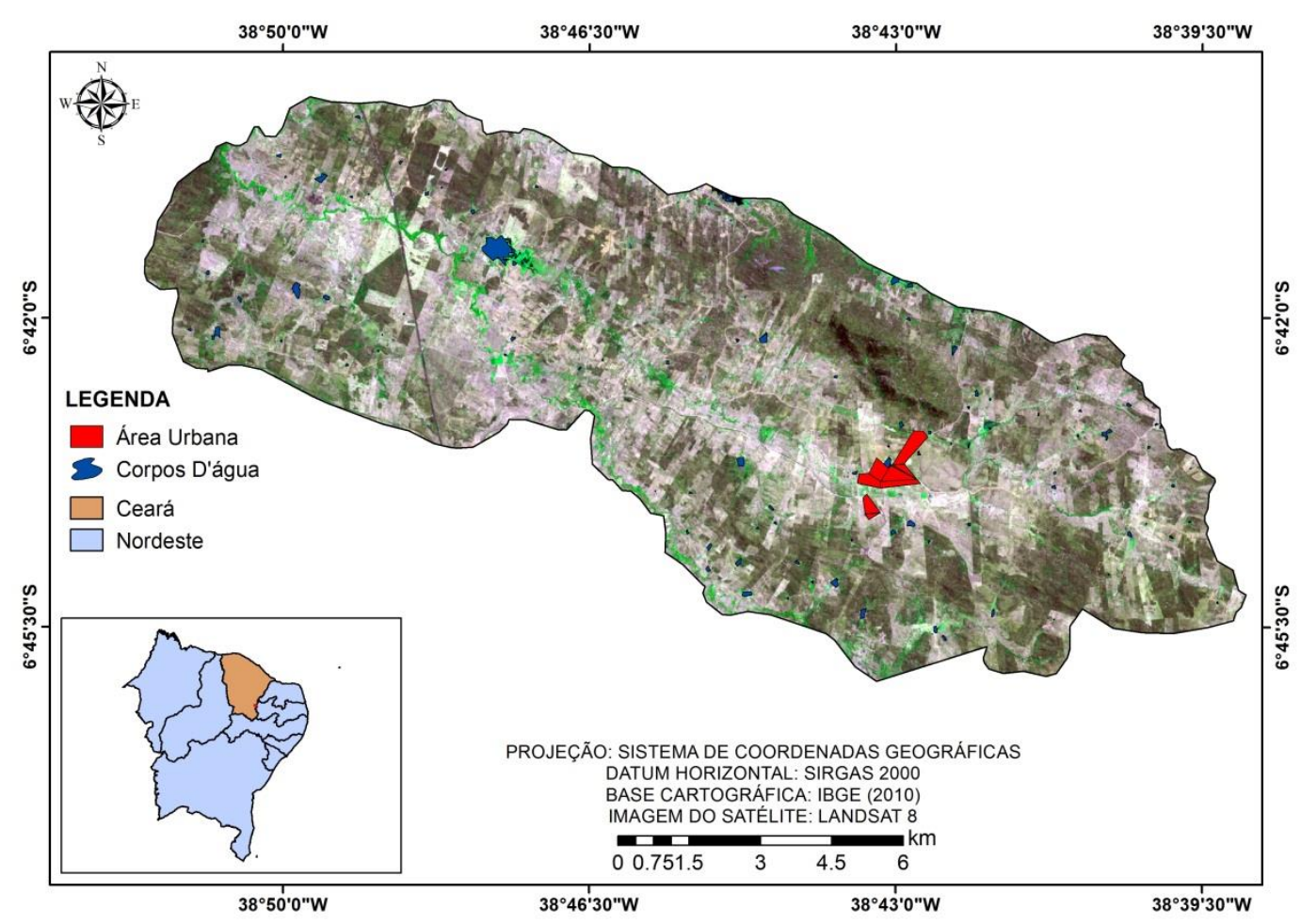

Figura 01. Localização da área de estudo.

Fonte: Autores (2018).

Segundo a classificação de Köppen-Geiger, o município de Baixio possui um clima tropical quente semiárido (BSh), caracterizado por altas temperaturas, elevada taxa de evapotranspiração, distribuição irregular da precipitação pluvial na escala espacial e no tempo, assim como períodos prolongados de estiagem (CRISPIM et al., 2017; IPECE; FUNCEME, 2013).

A temperatura média anual fica entre 26 a $28^{\circ} \mathrm{C}$, com baixa variação em suas médias mensais (CRISPIM et al., 2017), a precipitação pluviométrica anual registrada no município é superior a $900 \mathrm{~mm}$ (SILVA et al., 2016), tendo duas estações bem definidas, uma chuvosa e outra seca.

Os tipos de solos predominantes no município são uma associação dos Luvissolos (solos rasos a pouco profundos, com horizonte B textural de cores vivas e argila de atividade alta), Argissolos (solos de profundidade variável, moderadamente ácido e de cores avermelhadas ou amareladas) e Neossolos (solos de baixa profundidade, pequena retenção de água e elevada vulnerabilidade à inundação) (EMBRAPA, 2013; IPCE, 2013).

A formação florestal no município é constituída pela caatinga arbustiva densa com trechos de floresta caducifólia espinhosa (LEAL et al., 2003), composta pelas seguintes espécies vegetais: Amburana cearensis, Spondias tuberosa, Aspidosperma pyrifolium, Caesalpinia spp., Mimosa spp., Jatropha spp., Acacia spp. (CRISPIM et al., 2014; LEAL et al., 2003). No que tange a floresta caducifólia espinhosa (Caatinga Arbórea), consiste em uma formação vegetal característica da região Nordeste do 
USO E OCUPAÇÃO DO SOLO E SUA ASSOCIACCÃO

COM A TEMPERATURA DA SUPERFÍCIE

TERRESTRE NO MUNICÍPIO DE BAIXIO - CE

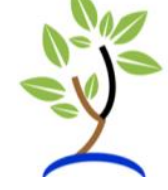

país, composta por arbustos e de árvores espontâneas, densa, baixa, de característica seca, de folhas pequenas, que caducam no verão e com raízes penetrantes e desenvolvidas, por exemplo, plantas xerófilas (CRISPIM et al., 2014; KAZMIERCZAK et al.,1996).

\section{Obtenção das imagens de satélite}

Para a elaboração dos mapas foram utilizadas imagens orbitais do sensor TM do satélite Landsat-5, em que foram selecionadas as bandas 3, 4 e 5, e do sensor OLI, as bandas 4, 5 e 6 , do Landsat 8 , com resolução espacial de 30 metros $(\mathrm{m})$, para 0 estudo do uso e ocupação do solo (UOS).

As imagens do satélite Landsat 5 e 8 foram adquiridas através da base de dados do site do serviço geológico dos Estados Unidos (USGS).

Para estimar a temperatura da superfície terrestre (TST) na área de estudo, foram utilizadas duas imagens multiespectrais (bandas do infravermelho termal), correspondente 6 e 10, com resolução de 30 m, dos satélites Landsat 5 e 8, por meio dos sensores TIRS (Thermal Infrared Sensor).

Para escolha das imagens que foram utilizadas na pesquisa, considerou-se como critério a baixa incidência de nebulosidade na área de estudo, que ocorre no período de estiagem. Assim sendo, as imagens selecionadas foram das seguintes datas: 28/08/1986, 20/07/1996, 25/09/2006 e 22/08/2016. Os procedimentos referidos em seguida foram executados com ajuda dos softwares ENVI@ 4.7 e ArcGIS 10.1.

\section{Temperatura de superfície}

Para a extração da Temperatura de Superfície na área de estudo foi realizado a conversão do nível de cinza (NC) para radiância e posteriormente determinou a temperatura em Kelvin, conforme as equações I e II, de acordo com a metodologia de Chander et al. (2009). Os sensores termais estão posicionados para detectar a radiação e são muito utilizados para determinação da TST (GUSSO; FONTANA; GONÇALVES, 2007).

$$
L_{\lambda}=M\left\llcorner Q_{c a l}+A_{L}\right.
$$

Sendo $L \lambda$ é a Radiância Espectral do sensor de abertura em Watts/( $\left.\mathrm{m}^{2} \mathrm{sr} \mu \mathrm{m}\right)$; $\mathrm{ML}$ é Fator multiplicativo de redimensionamento da banda termal; Qcal é o Valor quantizado calibrado pelo pixel na banda termal e AL é o Fator de redimensionamento aditivo específico da banda termal. 
USO E OCUPAÇÃO DO SOLO E SUA ASSOCIACCÃO

COM A TEMPERATURA DA SUPERFÍCIE

TERRESTRE NO MUNICÍPIO DE BAIXIO - CE

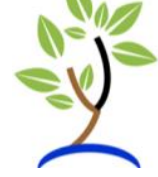

Logo após a transformação dos valores em radiância, utilizou se a equação II com a finalidade de extrair a temperatura em Kelvin.

$$
\mathrm{T}=\mathrm{K} 2 / \ln \left(\mathrm{K}_{1} / \mathrm{L} \mathrm{Y}^{+1}\right)
$$

Em que: $\mathrm{T}=$ Temperatura efetiva no satélite em Kelvin; $\mathrm{K} 2$ = Constante de calibração; $\mathrm{K} 1$ = Constante de calibração. K1 e k2 são constantes de conversão térmica para os satélites do Landsat 5 e 8, conforme a tabela 1.

Após a aquisição da temperatura em Kelvin, o valor da temperatura foi subtraído por 273,15 (zero absoluto) para se determinar a temperatura em graus Celsius $\left({ }^{\circ} \mathrm{C}\right)$. $\mathrm{O}$ mesmo processo foi realizado para o satélite Landsat 5 e 8.

Tabela 1: Parâmetros utilizados para a análise da temperatura de superfície

\begin{tabular}{c|c|c}
\hline & Landsat 5 & Landsat 8 \\
\hline $\boldsymbol{M}_{\boldsymbol{L}}$ & 0.055 & 0.00033420 \\
\hline $\boldsymbol{A}_{\boldsymbol{L}}$ & 1.18243 & 0.10000 \\
\hline $\mathbf{Q c a l}$ & Banda 6 & Banda 10 \\
\hline K1 & 607.76 & 774.8853 \\
\hline K2 & 1260.56 & 1321.0789 \\
\hline \multicolumn{2}{|c}{ Fonte: Adaptado de Coelho; Correa (2013). }
\end{tabular}

\section{Uso do Solo}

$\mathrm{Na}$ fase de processamento, utilizou a composição falsa cor RGB 5,4,3 e 6,5,4 para os satélites Landsat 5 e 8, respectivamente para a análise do uso do solo. A classificação do uso do solo foi realizada por meio da técnica da Máxima Verossimilhança (MAXVER) (BARROS et al., 2013). Este método consiste na classificação pixel a pixel da imagem e o algoritmo calcula a probabilidade de um determinado pixel pertencer a uma nova classe (LEITE; ROSA, 2012). Nesta pesquisa foram utilizadas as seguintes classes: Área Antropizada, Corpo Hídrico e Floresta.

Para a validação da classificação do uso e cobertura do solo utilizou-se como parâmetro o índice Kappa, usualmente empregado em diversos estudos, por exemplo, QUEIROZ et al., 2017; DEMARCHI et al., 2011; BARBOSA et al., 2011; CONGALTON; GREEN, 1999.

Este índice mostra a probabilidade de um pixel ser classificado corretamente em relação à classificação incorreta, assim os valores de Kappa alcançados neste estudo foram aferidos com base na classificação determinada por Landis; Koch, (1977) (Tabela 1). 
USO E OCUPAÇÃO DO SOLO E SUA ASSOCIACCÃO

COM A TEMPERATURA DA SUPERFÍCIE

TERRESTRE NO MUNICÍPIO DE BAIXIO - CE

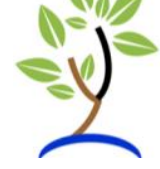

Tabela 2. Critérios de classificação ligados ao índice estatístico de Kappa

\begin{tabular}{c|c}
\hline Valor de Kappa & Grau de desempenho \\
\hline$<0,0$ & Péssima \\
\hline $0,0-0,2$ & Ruim \\
\hline $0,40-0,60$ & Razoável \\
\hline $0,40-0,60$ & Boa \\
\hline $0,60-0,80$ & Muito boa \\
\hline $0,80-1,0$ & Excelente \\
\hline
\end{tabular}

Fonte: Landis; Koch, (1977).

Posteriormente, para avaliar os resultados a serem obtidos por meio desta classificação serão confeccionados mapas temáticos no software ArcGIS 10.1, em que serão elaborados por meio da análise em tela da cena determinada. Assim, serão confeccionados mapas de referência para os períodos observados neste estudo.

\section{RESULTADOS E DISCUSSÃO}

Os valores obtidos na classificação para cada uma das classes, em seus respectivos períodos, são apresentados na Tabela 2. Nesta, constata-se que a classe com maior representação na área territorial do município em estudo é a área antropizada, esta classe teve um crescimento constante, apresentando variação de 128,89 km² a 134,37 $\mathrm{km}^{2}$. Posteriormente, a esta classe vem à floresta, que varia entre $11,29 \mathrm{~km}^{2}$ a 15,76 $\mathrm{km}^{2}$, e por fim, a classe corpo hídrico com variação de $0,62 \mathrm{~km}^{2}$ a 1,72 km². Enquanto, na Figura 2 é ilustrada a representação de cada uma destas dentro da extensão territorial do município de Baixio-CE.

Tabela 3. Tipos de classes de uso do solo, área e proporção

\begin{tabular}{c|c|c|c|c|c|c|c|c}
\hline \multirow{2}{*}{ Classes } & \multicolumn{2}{|c|}{1986} & \multicolumn{2}{c|}{1996} & \multicolumn{2}{c|}{2006} & \multicolumn{2}{c}{2016} \\
\cline { 2 - 9 } & $\mathrm{km}^{2}$ & $\%$ & $\mathrm{~km}^{2}$ & $\%$ & $\mathrm{~km}^{2}$ & $\%$ & $\mathrm{~km}^{2}$ & $\%$ \\
\hline Água & 1,63 & 1,11 & 1,72 & 1,17 & 1,27 & 0,87 & 0,62 & 0,42 \\
\hline Floresta & 15,76 & 10,77 & 13,83 & 9,45 & 14,09 & 9,63 & 11,29 & 7,72 \\
\hline Área Antropizada & 128,89 & 88,11 & 130,72 & 89,37 & 130,91 & 89,50 & 134,37 & 91,86 \\
\hline Total & 146,28 & 100 & 146,27 & 100 & 146,28 & 100 & 146,28 & 100 \\
\hline
\end{tabular}

Fonte: Dados da pesquisa (2018).

Para analisar o comportamento das classificações das imagens necessitou da confecção de mapas de referências, no qual foram obtidos por meio da análise em tela da área pesquisada, por meio das imagens de satélites para os respectivos anos estudados (Figura 2), assim foram determinadas três classes de uso do solo. 
USO E OCUPAÇÃO DO SOLO E SUA ASSOCIAÇÃO

COM A TEMPERATURA DA SUPERFÍCIE

TERRESTRE NO MUNICÍPIO DE BAIXIO - CE

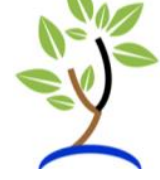

Observa-se por meio da espacialização do uso e ocupação do solo ilustrado na Figura 2, uma mudança no comportamento das classes estudadas entre os anos de $1986 \mathrm{e}$ 2016, em que se constata uma dinâmica de redução das classes floresta e corpo hídrico no período observado, sendo substituídas pela classe área antropizada.

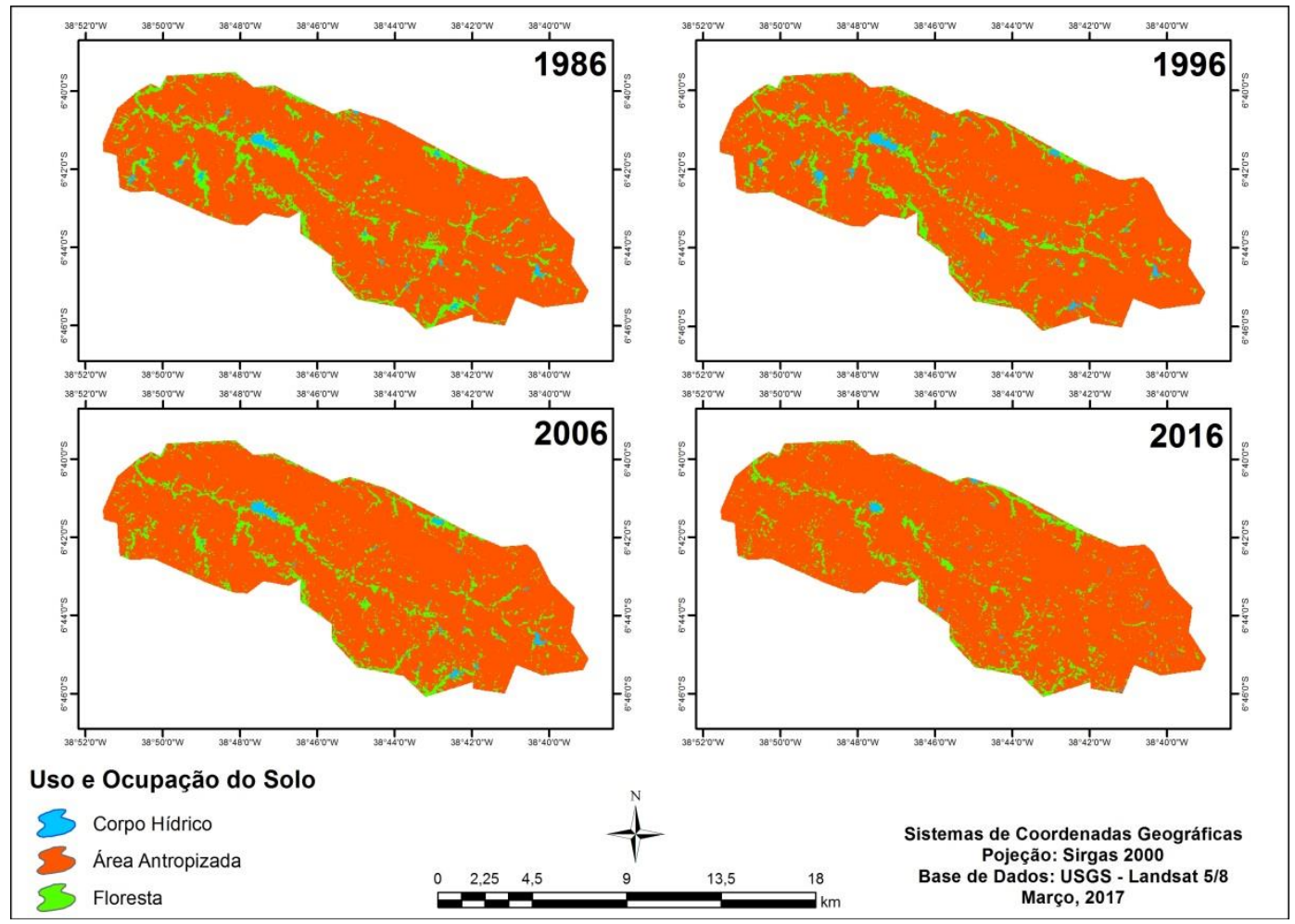

Figura 2. Uso e cobertura do solo (UCS) do município de Baixio-CE. Fonte: Autores (2018).

Este cenário pode ser resultante da utilização inapropriada dos recursos naturais que colaboram para alterações destas classes. Pois, no semiárido brasileiro, o desenvolvimento econômico está alicerçado, especialmente, na extração dos recursos naturais (SILVA et al., 2018). Todavia, isso não consiste em um obstáculo em si, à excepção quando os recursos são utilizados sob um princípio de otimização em pequeno período, sem propósitos de sustentabilidade em um longo período (SILVA et al., 2018).

No município em estudo existem diversos habitantes que utilizam de forma direta ou indiretamente os recursos naturais da Caatinga para sobreviver ou atender suas necessidades. Atualmente, o aproveitamento dos recursos da Caatinga baseia-se em processos meramente extrativistas para exploração de produtos de origem pastoril, agrícola e madeireiro (fabricação de carvão vegetal, extração de madeiras diversas para lenha e construção de cercas, além de atividades com a carnaúba) (IBGE, 2010).

A formação florestal do município vem sendo substituída pelas atividades ligadas a agricultura de subsistência (milho, feijão, arroz e batata) e a pecuária extensiva, principalmente a bovinocultura. Deste modo, as espécies vegetais têm sido cada vez 
USO E OCUPAÇÃO DO SOLO E SUA ASSOCIAÇÃO

COM A TEMPERATURA DA SUPERFÍCIE

TERRESTRE NO MUNICÍPIO DE BAIXIO - CE

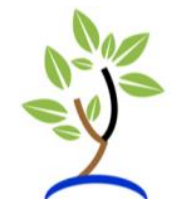

mais devastadas pela presença incessante destas duas atividades. Segundo Sousa et al. (2008) as atividades associadas a agropecuária, por exemplo, o desmatamento e a queima da floresta para formação de pastagens e a agricultura de sequeiro foram intensamente influenciadas pelo modelo de desenvolvimento econômico introduzido a partir do período de colonização, prejudicando de modo desfavorável a sustentabilidade dos recursos naturais.

O desmatamento consiste em uma das principais ações que ocasiona a degradação ambiente na região Nordeste, sempre ligado às atividades do setor agropecuário, assim como da produção agrícola, atividade industrial e aumento populacional (OLIVEIRA, 2017; SOUSA et al., 2008). O desmatamento e as queimadas causam diversas implicações negativas, por exemplo, a redução da biodiversidade, deterioração do solo, erosão do solo, aumento do escoamento superficial, entre outros fatores (SOUSA et al., 2008).

Poleto (2009) assegura que a degradação do meio ambiente está associada com atividades como, por exemplo, a agricultura, pois, nesta acontece à retirada da cobertura vegetal, ocasionando vários problemas para o solo, a saber, susceptibilidade aos processos erosivos e compactação do solo. Assim como, a redução da fertilidade natural do solo, acumulação e transporte de sedimentos para os corpos hídricos, ocasionando o assoreamento.

Estas atividades causam sérios problemas de modificação e degradação ambiental resultante de uma exploração insustentável e de um manejo inadequado dos seus recursos naturais, onde são explorados de forma predatória. Estas ações antrópicas concatenado às peculiaridades climáticas, tornam ainda mais instável o equilíbrio ecológico, com consequências negativas para os recursos ambientais e para a condição de vida dos habitantes.

A população desta região necessita de novas formas de pensar, sentir e agir no ambiente no qual estão inseridos. Portanto, é imprescindível uma mudança de concepção sobre a realidade local, buscando a experimentação de alternativas de produção apropriada com as condições naturais da região. A construção de novas possibilidades de desenvolvimento deve estar articulada às medidas de gestão ambiental sustentável concatenado as iniciativas sociais que resultam em melhoria das condições de vida.

Diante desse panorama, uma alternativa seria a realização de investimentos em pesquisas, com a finalidade de conhecer melhor as potencialidades dos recursos naturais locais que poderão auxiliar na definição de ações para um melhor aproveitamento econômico através de tecnologias sociais adaptadas às condições naturais do ambiente e que possam mitigar a ações de degradação deste bioma.

A formulação e realização de projetos de manejo sustentável dos recursos naturais, de uma produção apropriada e da melhoria das condições socioculturais da população sertaneja pode ser uma opção para atenuar o processo de degradação ambiental que ocorre no município. Além disso, é importante inserção de técnicas florestais no cotidiano do agricultor, a saber, o uso de sistemas agroflorestais, visando uma maior ampliação espacial entre atividades florestais e agrícolas. 
USO E OCUPAÇÃO DO SOLO E SUA ASSOCIAÇÃO

COM A TEMPERATURA DA SUPERFÍCIE

TERRESTRE NO MUNICÍPIO DE BAIXIO - CE

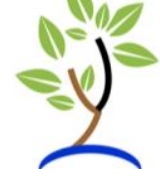

Assim, a partir da utilização sustentável dos recursos naturais existentes no bioma Caatinga e do manejo adequado da sua biodiversidade poderá impulsionar o desenvolvimento da região. Esta ação é uma forma de amenizar as práticas não apropriadas à realidade local, caracterizada pela excessiva exploração dos recursos naturais. A Tabela 3 apresenta os valores do coeficiente de Kappa e grau de desempenho com base em Landis e Koch (1997) para cada um dos períodos estudados.

Tabela 4. Desempenho do índice Kappa e a qualidade da classificação para os períodos analisados

\begin{tabular}{c|c|c}
\hline Período & Índice de Kappa & Grau de desempenho \\
\hline $28 / 08 / 1986$ & 0,93 & Excelente \\
\hline $20 / 07 / 1996$ & 0,83 & Excelente \\
\hline $25 / 09 / 2006$ & 0,53 & Boa \\
\hline $22 / 08 / 2016$ & 0,50 & Boa \\
\hline \multicolumn{2}{r}{} \\
\end{tabular}

Conforme se verifica na Tabela 3 a qualidade da classificação executada obteve um índice de Kappa excelente para os anos de 1986 e 1996, validando a escolha pelo classificador da Máxima Verossimilhança (BARROS et al., 2013; BARBOSA et al., 2011) para esta forma de análise. Todavia, para os anos de 2006 e 2016, o índice de qualidade de classificação alcançou um desempenho bom.

Com base na Figura 3, constata-se que a temperatura da superfície da terra no município de Baixio apresentou um aumento significativo no período observado. 


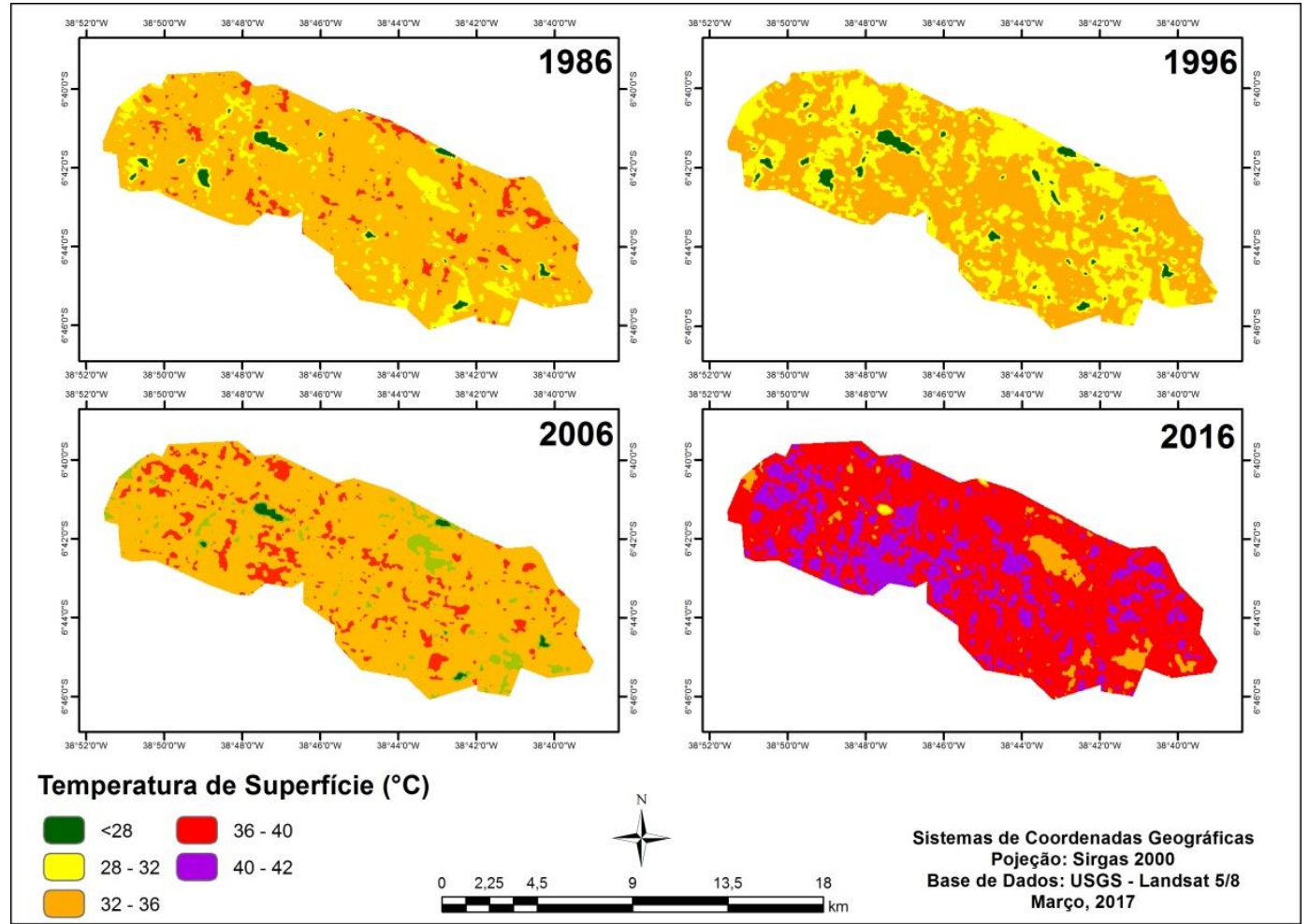

Figura 3. Temperatura de superfície terrestre do município de Baixio - CE Fonte: Autores (2018).

Assim, os anos com as maiores médias registradas foram 2006 e 2016, porém, com maior destaque para 2016, em que a predominância das temperaturas ficou entre os intervalos de 36 a $40{ }^{\circ} \mathrm{C}$ e 40 e $42^{\circ} \mathrm{C}$., em geral, esta variação da TST pode ser resultante da modificação da superfície natural, por exemplo, a retirada da floresta para atividades extrativistas, assim como a substituição da floresta pela agricultura de subsistência e a pecuária extensiva, atividades que contribuem para redução da cobertura vegetal, aumentando a exposição do solo.

Segundo Costa et al. (2016) o solo exposto, ou seja, desnudo possuem elevações térmicas, por conseguinte, altas temperaturas por causa da ausência da cobertura vegetal na área e da exposição do solo à radiação solar. Assim, conforme estes autores a cobertura florestal ameniza a absorção e perdas de calor, possuindo temperaturas menores que as áreas antropizadas.

As menores médias da TST foram registradas no ano de 1996, em especial nas classes corpo hídrico e floresta. Assim, contata-se que as temperaturas menores que $28^{\circ} \mathrm{C}$ ou entre 28 e $32^{\circ} \mathrm{C}$ foram registradas em áreas com cobertura vegetal, por exemplo, floresta ciliar ou em locais próximos de corpos hídricos, a saber, reservatório artificial ou natural, lago, lagoa ou aquífero subterrâneo. Deste modo, isto pode ser constatado quando confrontar a Figura 2 que representa o uso e ocupação do solo com a Figura 3 que especializa a TST para o período observado no município em estudo. 
USO E OCUPAÇÃO DO SOLO E SUA ASSOCIACCÃO

COM A TEMPERATURA DA SUPERFÍCIE

TERRESTRE NO MUNICÍPIO DE BAIXIO - CE

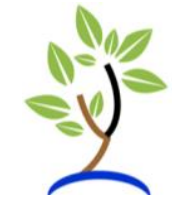

\section{CONCLUSÃO}

Por meio da análise do uso e ocupação do solo realizado no município de Baixio $\mathrm{CE}$, durante o período analisado constatou-se que a classe área antropizada apresentou um crescimento contínuo. Enquanto, as classes corpo hídrico e floresta tiveram uma redução de suas porções na extensão territorial do município estudado, conforme observado nos mapas e os dados de proporções das áreas para cada classe.

No que tange à temperatura de superfície, observou-se um aumento considerável no período estudado. Além disso, os anos que apresentaram as maiores médias observadas foram 2006 e 2016, contudo, com maior evidência para 2016, em que as temperaturas predominantes ficaram entre 36 a $40^{\circ} \mathrm{C}$ e 40 e $42^{\circ} \mathrm{C}$.

De modo geral, tanto o uso e ocupação do solo como a variação da TST possivelmente estão sofrendo influências ocasionadas pelas atividades antrópicas realizadas no território do município, causando modificações na paisagem natural.

\section{REFERÊNCIAS}

ALMEIDA, A. J. P, et al.. Relação entre o Índice de Vegetação e a Temperatura de Superfície na estimativa e identificação das ilhas de calor na cidade de Maceió-AL. Simpósio Brasileiro de Sensoriamento Remoto - SBSR, 17., 2015, João Pessoa. Anais... João Pessoa: INPE, 2015.

BARBOSA, A. P.; CAMPOS, S.; ZIMBACK, C. R. L.; XAVIER, Z. B.. Comparação de métodos de classificação de imagens na identificação de áreas cultivadas com citros. Revista Energia na Agricultura, Botucatu, vol. 26, n.3, p.14-25, 2011.

BARROS, K. O. et al.. Análise temporal das classes de uso e ocupação da terra na bacia hidrográfica do Rio Vieira, Montes Claros, Minas Gerais. Revista Agrogeoambiental, Pouso Alegre, v. 5, n. 2, caderno I, p. 43-54, ago. 2013.

BEZERRA, J. M.; MOURA, G. B. de A.; SILVA, B. B. da; LOPES, P. M. O.; SILVA, E. F. de F.. Parâmetros biofísicos obtidos por sensoriamento remoto em região semiárida do estado do Rio Grande do Norte, Brasil. Revista Brasileira de Engenharia Agrícola e Ambiental, v.18, n.1, p.73-84, 2014.

CHANDER, G.; MARKHAM, B. L.; HELDER, D. L.. Summary of current radiometric calibration coefficients for Landsat MSS, TM, ETM+, and EO-1 ALI sensors. Remote Sensing of Environment, v.113, p. 893-903, 2009.

COELHO, A. L. N. CORREA, W. S. C. Temperatura de Superfície Celsius do Sensor Tirs/Landsat-8: Metodologia e Aplicações. Rev. Geogr. Acadêmica, v.7, n.1, p. 31-45, 2013. 
USO E OCUPAÇÃO DO SOLO E SUA ASSOCIACCÃO

COM A TEMPERATURA DA SUPERFÍCIE

TERRESTRE NO MUNICÍPIO DE BAIXIO - CE

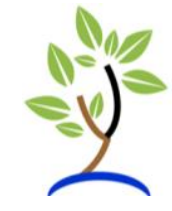

COELHO, V. H. R.; MONTENEGRO, S. M. G. L.; ALMEIDA, C. das N.; LIMA, E. R. V. de; RIBEIRO NETO, A.; G. S. S. de MOURA.. Dinâmica do uso e ocupação do solo em uma bacia hidrográfica do semiárido brasileiro. Revista Brasileira de Engenharia Agrícola e Ambiental, v.18, n.1, p.64-72, 2014.

CONGALTON, R.G.; GREEN, K.. Assessing the Accuracy of Remotely Sensed Data: Principles and Practices. Nova York, Boca Raton: Lewis Publishers, 1999. 137 p.

COSTA, A. M. S. da; BEZERRA, P. E. S.; OLIVEIRA, R. S.. Análise da temperatura da superfície terrestre associada à dinâmica do uso e ocupação do solo nos municípios de Belém e Ananindeua, Pará, Brasil. Simpósio de Geotecnologias no Pantanal, 6., 2016, Cuiabá. Anais... Cuiabá: Embrapa Informática Agropecuária/INPE, 2016. p. $957-967$

CRISPIM, D. L.; ANDRADE, S. O. de; MENESES, J. A. D. de; CHAVES, A. D. C. G.; BORGES, M. da G. B.. Impactos ambientais decorrentes do uso e ocupação desordenada do espaço urbano: um estudo de caso da cidade de Baixio/CE. Revista Verde de Agroecologia e Desenvolvimento Sustentável, v 9., n.4, p. 44 - 49, out-dez, 2014.

CRISPIM, D. L.; MENESES, J. A. D. de.; VIEIRA, A. S. de A.; ARAÚJO, L. M. de.; CRUZ, J da S.; SILVA, J. I. S.. Levantamento quali-quantitativo da arborização urbana da cidade de Baixio - CE. Revista Principia, João Pessoa, [S.I.], n. 36, p. 99-106, set. 2017. ISSN 2447-9187. Disponível em: <http://periodicos.ifpb.edu.br/index.php /principia/article/view/972>. Acesso em: 03 maio 2018.

DEMARCHI, J. C.; PIROLI, E. L.; ZIMBACK, C. R. L.. Análise temporal do uso do solo e comparação entre os índices de vegetação NDVI e SAVI no município de Santa Cruz do Rio Pardo - SP usando imagens Landsat-5. Ra'e ga (UFPR), v. 21, p. 234271, 2011.

EMBRAPA. Empresa Brasileira de Pesquisa Agropecuária. Sistema brasileiro de classificação de Solos. 3. ed. rev. ampl. - Brasília, DF: Embrapa, 2013. 353p.

FERREIRA, A. B.; PEREIRA FILHO, W.. Análise do uso e cobertura da terra de três sub-bacias hidrográficas - Rio Grande do Sul/Brasil. Revista Brasileira de Geografia Física, Recife, v. 2, n.03, set-dez, p. 20-36, 2009.

FERREIRA, B.; ZIMMERMANN, D. M.; CRISPIM, L. C.; M. F. FLACH; VIEIRA, C. A. O.. Análise sazonal das temperaturas superficiais do estado de Santa Catarina entre os anos de 2000 e 2010. Congresso Brasileiro de Cartografia, 17., 2017, Rio de Janeiro. Anais... Rio de Janeiro: SBC, 2017. p. 933-937.

FLORENZANO, T.G.. Iniciação em Sensoriamento Remoto. 3. ed. São Paulo: Oficina de Texto, 2011. 
USO E OCUPAÇÃO DO SOLO E SUA ASSOCIACCÃO

COM A TEMPERATURA DA SUPERFÍCIE

TERRESTRE NO MUNICÍPIO DE BAIXIO - CE

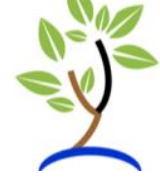

GUSSO, A.; FONTANA, D. C.; GONÇALVES, G. A.. Mapeamento da temperatura da superfície terrestre com uso do sensor AVHRR/NOAA. Pesquisa Agropecuária Brasileira, Brasília, v.42, n.2, p.231-237, 2007.

IBGE. Instituto Brasileiro de Geografia e Estatística. Censo Demográfico 2010. Rio de Janeiro: IBGE, 2010. Disponível em: <http://cod.ibge.gov.br/el8>. Acesso em: 08 jan. 2018.

IPECE. Instituto de Pesquisa e Estratégia Econômica do Ceará. Perfil Básico Municipal 2013: Baixio. 2013. Disponível em: <www.ipece.ce.gov.br/ publicacoes/ perfil_ba-sico/pbm-2013/Baixio.pdf>. Acesso em: 08 jan. 2018.

KAZMIERCZAK, M.L. et al.. Identificação de área de preservação florestal, através de técnicas de geoprocessamento: abordagem preliminar. In: Simpósio Brasileiro de Sensoriamento Remoto, 8., 1996, Salvador. Anais... Salvador: INPE, 1996. p. 181185.

LANDIS, R.; KOCH, G. G.. The measurement of observer agreement for categorical data. Biometrics, v.33, n.1, p.159-174,1977.

LEAL, I.R. et al.. Ecologia e conservação da caatinga. Recife: Ed. Universitária da UFPE, 2003. 822 p.

LEITE, E. F.; ROSA, R.. Análise do uso, ocupação e cobertura da terra da bacia hidrográfica do Rio Formiga, Tocantins. Revista Eletrônica de Geografia, v.4, n.12, p. 90-106, 2012.

MATSUSHITA, B.; XU, M.; FUKUSHIMA, T.. Characterizing the changes in landscape structure in the Lake Kasumigaura, Japan using a high-quality GIS dataset. Ladscape and Urban Planning, v.78, p.241-250, 2006.

MASHIKI, M. Y.; CAMPOS, S.. Influência do uso e ocupação do solo na temperatura aparente da superfície no município de Botucatu/SP. Energ. Agric., Botucatu, v. 28, n.3, p.143-149, jul.-set., 2013.

MENDOZA, M. E.; GRANADOS, E. L.; GENELETTI, D.; PÉREZ-SALICRUP, D. R.; SALINAS, V.. Analysing land cover and land use change process at watershed level: A multitemporal study in the Lake Cuitzeo Watershed, Mexico (1975-2003). Applied Geography, v.31, p.237-350, 2011.

OLIVEIRA, F. L. S.; SILVA, C. A.; SILVA, E. E. S.; ALMEIDA, L. Q.. Classificação dos diferentes tipos de uso do solo nos sistemas ambientais do município de Pacoti, Ceará. Simpósio Brasileiro de Geografia Física Aplicada, 17., 2017, Congresso Nacional de Geografia Física, 1., 2017, Campinas. Anais... Campinas: Instituto de Geociências - UNICAMP, 2017. 
USO E OCUPAÇÃO DO SOLO E SUA ASSOCIACCÃO

COM A TEMPERATURA DA SUPERFÍCIE

TERRESTRE NO MUNICÍPIO DE BAIXIO - CE

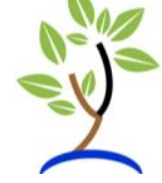

PEREIRA, C. C.; MARIANO, Z. F.; WACHHOLZ, F.; CABRAL, J. B. P.. Análise da temperatura de superfície e do uso da terra e cobertura vegetal na bacia Barra dos Coqueiros. Revista Geonorte, Edição Especial 2, v.2, n.5, p.1243 - 1255, 2012.

PONZONI, F. J.; SHIMABUKURO, Y. E.; KUPLICH, T. M. Sensoriamento Remoto No Estudo da Vegetação. 2. ed. São Paulo: Ofícina de Textos, 2012. 176p.

POLETO, C. Introdução ao gerenciamento ambiental. 1. ed. Rio de Janeiro: Interciencia, 2010. 336p.

QUEIROZ, T. B.; SOUSA, R. dos S.; BALDIN, T.; BATISTA, F. de J.; MARCHESAN, J.; PEDRALI, L. D.; PEREIRA, R. S.. Avaliação do desempenho da classificação do uso e cobertura da terra a partir de imagens Landsat 8 e Rapideye na região central do Rio Grande do Sul. Geociências, São Paulo, v. 36, n. 3, p. 569 - 578, 2017.

SILVA, A.M.; R.M. SILVA; B.B. SILVA.. Determinação de Temperatura da Superfície e Estimativa do Saldo de Radiação e Evapotranspiração Usando Imagens Landsat e Dados Observados. Revista Brasileira de Cartografia, v. 67/6, p. 1203-1216, 2015.

SILVA, E. B. da.; MACÊDO, K. G. de.; FEITOSA, A. K.; ARAÚJO NETO, J. R. de. Variação espacial da precipitação das cidades do centro do sul do estado do Ceará, Brasil. Revista Perspectiva Online, v.6, n.16, p. 27-33, 2016. Disponível em:< http://www.seer.perspectivasonline.com.br/index.php/exatas_e_engenharia/article/vi ew/640/834>. Acesso em: 17 jan. 2018.

SILVA, J. L. C.; VIDAL, C. A. S.; BARROS, L. M.; FREITA, F. R. V.. Aspectos da degradação ambiental no Nordeste do Brasil. R. gest. sust. ambient., Florianópolis, v. 7, n. 2, p.180-191, abr./jun. 2018.

SILVA, J. M. O.. Uso do sensoriamento remoto para a estimativa da temperatura de superfície na Microbacia do Rio Granjeiro/Crato - Ceará. Geosaberes, Fortaleza, v. 6, n. 2, p. $130-144,2015$.

SOUSA, R. F. de; BARBOSA, M. P.; TERCEIRO NETO, C. P. C.; CARVALHO, A. de P.; LIMA, A N. Uso de geotecnologias no diagnóstico da degradação das terras do município de São João do Cariri-PB. Revista Caatinga, Mossoró, v.21, n.1, p.204-210, jan./mar., 2008.

VANZELA, L. S.; HERNANDEZ, F. B.; FRANCO, R. A. M.. Influência do uso e ocupação do solo nos recursos hídricos do Córrego Três Barras, Marinópolis. Revista Brasileira de Engenharia Agrícola e Ambiental, v.14, p.55-64, 2010.

Recebido em 10/07/2018 Aceito em 08/12/2018 